\title{
Exit from mitosis in D rosophila syncytial embryos requires proteolysis and cyclin degradation, and is associated with localized dephosphorylation
}

\author{
Tin Tin Su, Frank Sprenger, ${ }^{1}$ Paul J. DiGregorio, Shelagh D. Campbell, ${ }^{2}$ and Patrick H. O'Farrell ${ }^{3}$ \\ Department of Biochemistry and Biophysics, University of California at San Francisco, \\ San Francisco, California 94143-0448 USA
}

The cyclin proteolysis that accompanies the exit from mitosis in diverse systems appears to be essential for restoration of interphase. The early syncytial divisions of D rosophila embryos, however, occur without detectable oscillations in the total cyclin level or Colk1 activity. Nonetheless, we found that injection of an established inhibitor of cyclin proteolysis, a cyclin B amino-terminal peptide, prevents exit from mitosis in syncytial embryos. Similarly, injection of a version of D rosophila cyclin B that is refractory to proteolysis results in mitotic arrest. We infer that proteolysis of cyclins is required for exit from syncytial mitoses. This inference can be reconciled with the failure to observe oscillations in total cyclin levels if only a small pool of cyclins is destroyed in each cycle. We find that antibody detection of histone $\mathrm{H3}$ phosphorylation (PH3) acts as a reporter for Colk1 activity. A gradient of PH3 along anaphase chromosomes suggests local Colk1 inactivation near the spindle poles in syncytial embryos. This pattern of Cdk1 inactivation would be consistent with local cyclin destruction at centrosomes or kinetochores. The local loss of PH3 during anaphase is specific to the syncytial divisions and is not observed after cellularization. We suggest that exit from mitosis in syncytial cycles is modified to allow nuclear autonomy within a common cytoplasm.

[Key Words: Drosophila; mitosis; proteolysis; cyclin; histone H3]

Received January 21, 1998; revised version accepted March 16, 1998.

Although the past decade of cell cycle research has revealed a bewildering complexity of cell cycle control, one simple generalization has been supported by observations in a variety of experimental settings and in numerous species. The kinase activity of a cyclin:Cdk1 complex promotes mitosis, whereas the destruction of the cyclin partner and loss of kinase activity is associated with and is required for exit from mitosis. The early evidence for the requirement for cyclin destruction came from the production of deleted versions of cyclins that resisted mitotic destruction and arrested cells in mitosis (M urray et al . 1989; Gl otzer et al. 1991; Gallant and Nigg 1992; Surana et al. 1993; Sigrist et al. 1995a). However, some complexities have been introduced even into this most global generalization about the cell cycle. We now recognize that exit from mitosis is complex and that sequential destruction of many different regulators is likely to regulate distinct steps in the process, as detailed below.

Present addresses: 'Institut für Genetik, Universität zu Köln, D-50931 Köln, Germany; ${ }^{2}$ Department of Biological Sciences, University of Alberta, Edmonton, Alberta, Canada T6G 2E9.

${ }^{3}$ Corresponding author.

E-MAIL ofarrell@cg. .ucsf.edu; FAX (415) 502-5143/5145.
Mitosis may be considered a progression of discrete steps, with the ultimate goal being the equal division of the genomic contents. Among all mitotic phases, metaphase appears to represent the pinnacle of the mitotic state, with the spindle and the condensed chromosomes poised to segregate daughter genomes to the opposite poles of the cell. Rel ease from this state requires a ubiquitin-conjugating enzyme complex, APC (anaphase promoting complex; King et al. 1995), or cyclosome (Sudakin ${ }^{-}$et al. 1995) that targets cyclins and several other proteins for rapid proteolytic destruction (for review, see King et al. 1996a; N asmyth 1996). The original demonstration that the transition from metaphase requires proteolysis was shown in Xenopus egg extract experiments (Holloway et al. 1993). In these extracts, introduction of high level s of a target peptide for ubiquitinmediated proteolysis (containing the "destruction box") led to mitotic arrest, apparently by competing with endogenous substrates and interfering with their destruction (Holloway et al. 1993; van der Velden and Lohka 1993). Genetic analyses in yeast and biochemical experiments in mammalian systems established the universal requirement for ubiquitin-mediated proteolysis in exiting mitosis (for review, see King et al. 1996a; N asmyth 
1996). Although recent work focusing on the mechanisms that govern the exit from mitosis has identified new and important targets of APC-mediated proteolysis (e.g., see Cohen-Fix et al. 1996; Funabiki et al. 1996; Juang et al. 1997), the destruction of mitotic cyclins is still thought to represent a key and essential step in the return to an interphase state.

Removal of the destruction box sequences from the amino terminus of mitotic cyclins protects these proteins from mitotic degradation (Murray et al. 1989; Glotzer et al. 1991; Gallant and N igg 1992; Surana et al. 1993; Sigrist et al. 1995a). Stable cyclins block exit from mitosis, but stabilization of different cyclins blocks exit from mitosis at different steps (Sigrist et al. 1995a). In cellularized embryos of Drosophila melanogaster, stabilization of cyclin A (which has a mitotic function in Drosophila; Lehner and O'Farrell 1989, 1990; Knoblich and Lehner 1993) results in a metaphase-like arrest, with unsegregated sister chromosomes aligned at the metaphase plate. In contrast, mitotic arrest that results from stabilization of another mitotic cyclin, cyclin B, shows partially segregated condensed chromosomes and therefore, resembles early anaphase. Finally, stabilization of the third Drosophila mitotic cyclin, cyclin B3, results in a mitotic arrest with fully segregated chromosomes, thus resembling late anaphase/telophase. Similar to the situation with stabilized cyclin B3 of Drosophila, stable Btype cyclins can lead to postmetaphase arrest in budding and fission yeasts, Xenopus extracts, and mammalian cells (Holl oway et al. 1993; Sigrist et al . 1995a; Surana et al. 1993; Yamano et al. 1996). Interestingly, mitotic arrest that results from stabilization of each mitotic cycIin, A, B, and B3, in Drosophila embryos resembles the mitotic stage at which degradation of each cyclin is completed (Sigrist et al. 1995a). This observation has led to the proposal that sequential degradation of mitotic cyclins dictates the sequential order of some of the events at exit from mitosis (Sigrist et al. 1995a; for review, see Follette and O'Farrell 1997).

The precellular mitotic divisions of the Drosophila embryo appear to provide a striking counterpoint to the demonstrated importance of cyclin degradation and Cdk1 inactivation to cell cycle progression. Drosophila embryogenesis begins with 13 metasynchronous mitotic cycles within a syncytial cytoplasm (Foe et al. 1993). These cycles consist only of $S$ and $M$ phases, rely on maternally suppl ied activities, and do not requirezygotic gene expression. The first 10 syncytial cycles last $\sim 9 \mathrm{~min}$ each; subsequently, the cycles slow gradually, leading to a transition from maternal to zygotic control of the cell cycle in cycle 14. Previous studies indicate that cyclin levels and Cdk1 activity remain high during the first eight cycles (Edgar et al. 1994). Thereafter, oscillations of these key cell cycle regulators set in gradually, with the amplitude of the oscillation increasing in successive mitoses. Given that mitotic cyclin degradation and Cdk1 inactivation appear essential for exiting mitosis in all systems tested, we asked how syncytial cycles occur in the continuous presence of mitotic regulators.

Two cases in which cells exit mitosis without cyclin degradation are worth noting. In budding yeast, hctl/ cdh1 mutants are defective in the proteolysis of a mitotic cyclin, Clb2, and yet these cells are able to exit mitosis (Schwab et al. 1997). Second, in budding yeast cells that are arrested in mitosis because of spindle defects, a mutation in the phosphatase regulatory subunit CDC55 allowed the exit from mitosis (Minshull et al. 1996). In this case, exit from mitosis occurred in the presence of high cyclin levels, apparently by inhibitory phosphorylation of $\mathrm{Cdk} 1$. The absence of an inhibitory phosphate on Cdk1 in Drosophila syncytial cycles, however, argues against Cdk1 phosphorylation being responsible for exit from syncytial mitoses (Edgar et al. 1994). Instead, the fact that mitotic arrest follows cyclin stabilization in diverse experimental systems argues that $\mathrm{Cdk} 1$ inactivation by cyclin degradation may be the universal way of exiting mitosis in unperturbed cells.

In this report we demonstrate that despite the constant presence of cyclins and Cdk 1 activity, proteolysis and cyclin degradation appear to be essential for syncytial nuclei to exit mitosis. Using phosphorylation on the first serine of histone $\mathrm{H} 3(\mathrm{PH})$ as a reporter for $\mathrm{Cdk} 1$ activity, we present evidence for local changes in Cdk1 activity as syncytial nuclei exit mitosis. Such local changes are absent in the mitoses of the cellular blastoderm embryo in which mitotic cyclins are degraded completely at exit from mitosis. We propose that syncytial embryos experience local changes in Cdk1 activity through degradation of small, local pools of cyclins. We infer that in cellularized embryos global degradation of cyclins throughout the cell results in global changes such as the near-uniform loss of $\mathrm{PH} 3$ from chromosomes.

\section{Results and Discussion}

Injection of a destruction box peptide results in mitotic arrest in syncytial embryos

To assess the role of ubiquitin-mediated proteolysis in the syncytial cycles of Drosophila, we injected syncytial stage embryos with a known inhibitor of the process, an amino-terminal peptide of sea urchin cycl in B. This peptide contains the signal sequence for the ubiquitin-mediated degradation by the APC pathway, preceded by a T7 epitope tag. It is identical to the peptide used in an earlier demonstration of the importance of proteolysis for mitotic progression in Xenopus extracts (referred to as 13-110 in Holloway et al. 1993). Injection of the 13110 peptide into syncytial stage embryos and staining for the epitope tag reveal ed a gradient of peptide with the high point at the site of injection (not shown). Such injections blocked the nucl ear cycles in mitotic-like states near the point of injection, whereas nuclei distant from the site of injection progressed to the expected nuclear density (Fig. 1A). N early all injected embryos showed condensed chromosomes within a mitotic spindle near the point of injection, although not all arrested mitoses had the appearance of a normal metaphase. Such arrests were seen in syncytial embryos of all ages, including 

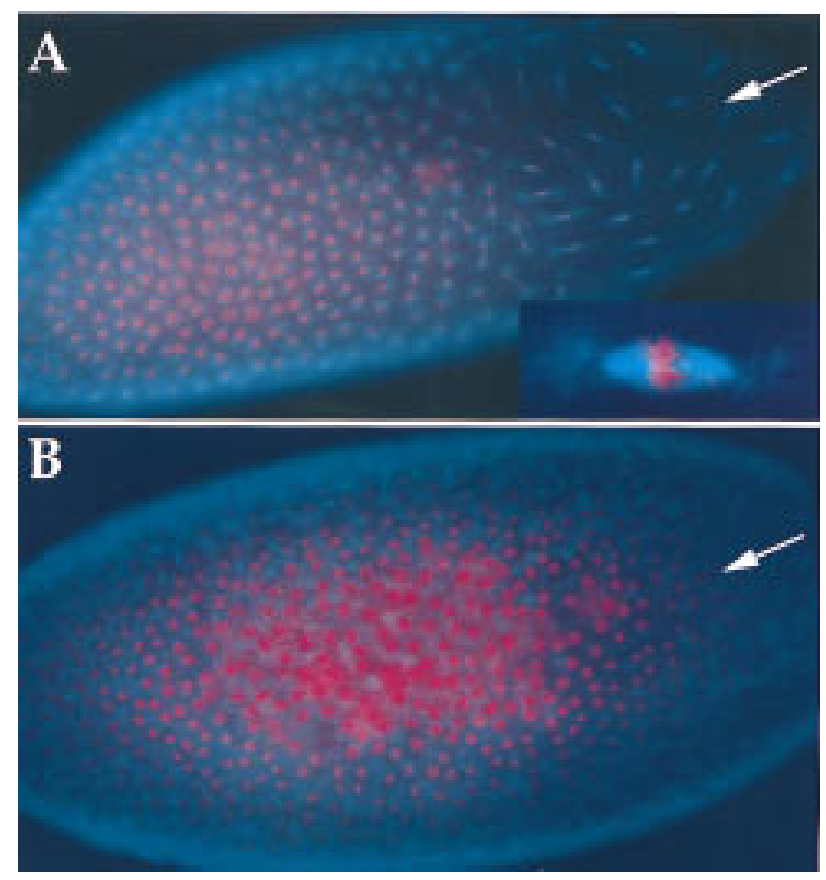

Figure 1. Amino-terminal peptide of cyclin B causes mitotic arrest. (A) Syncytial embryos (0- to 1-hr-old, before cycle 7) were injected with 13-110 peptide, fixed 30-45 min later, and stained for DNA (red) and $\beta$-tubulin (blue) to visualize microtubules. $\mathrm{N}$ uclei near the site of injection (arrow) are arrested in mitosis and show condensed chromosomes and mitotic spindles. In contrast, in the remainder of the embryo syncytial divisions have progressed such that nuclear density is higher and nuclei are not arrested in mitosis. An arrested mitotic figure is magnified fivefold and shown in the inset. (B) An embryo injected with the control peptide bearing two point mutations in the destruction box consensus sequence, 13-110*, shows no mitotic arrest. The site of injection is indicated with an arrow.

precycle 8 embryos, which ordinarily exhibit no detectable oscillations in cyclin levels or Cdk1 activity (Edgar et al. 1994). As a control, we injected a peptide in which the amino acids RAAL of the destruction box consensus sequence have been mutated to AARL to render it unrecognizable by the ubiquitin pathway (called 13-110* in Holloway et al. 1993). We found that the control peptide did not cause a mitotic arrest under similar conditions (Fig. 1B). We conclude that the presence of 13-110 interfered with mitotic progression, whereas 13-110* did not. The specificity implicates the destruction box in the inhibition of the nuclear cycles. The destruction box sequences target cyclin B for ubiquitination and proteolysis and the peptide containing this sequence interferes with these processes (van der Velden and Lohka 1993; King et al. 1996b). Therefore, our results suggest that APC-mediated ubiquitination, and presumably proteolysis, is required for exit from mitosis in the early syncytial cycles of Drosophila, although we cannot eliminate the possibility that ubiquitination might drive exit from mitosis without associated destruction of proteins.
Injection of a stable cyclin results in mitotic arrest in syncytial embryos

Studies in diverse systems identified mitotic cyclins as proteolytic substrates that must be removed to allow exit from mitosis. Recent work in yeast and A spergillus, however, has identified additional substrates that must be degraded to progress through events of mitosis (Pu and Osmani 1995; Cohen-Fix et al. 1996; Funabiki et al. 1996). These include proteins that regulate sister chromosome segregation and proteins associated with the mitotic spindle. Although the actual restoration of an interphase state is thought to usually require destruction of cyclins and inactivation of C dk1, neither cyclin levels nor Cdk1 activity oscillate in the early syncytial cycles. This rai ses the possi bility that it is the degradation not of cyclins but of other proteins that drives the exit from these mitoses. Thus, we assessed the importance of cyclin degradation to the syncytial cycles.

The importance of cyclin destruction to exiting mitosis has been demonstrated by the production of a mitotic arrest by stabilized cyclins when these proteins are introduced into cells or cycling Xenopus extracts (M urray et al. 1989; Gallant and Nigg 1992; Holl oway et al. 1993; Surana et al. 1993; Sigrist et al. 1995a). To test the importance of cyclin degradation to the early mitotic cycles, we prepared a stable derivative of Drosophila cyclin B protein for injection into syncytial embryos. In addition to truncations that removed sequences inferred to represent the destruction box (Sigrist et al. 1995a; Sprenger et al. 1997), a fusion of gl utathione S-transferase (GST) to the amino terminus of full-length Drosophila cyclin B proved to be stable. GST-cyclin B reconstituted kinase activity upon addition of in vitrotranslated Drosophila Cdk1 (Campbell et al. 1995), and incubation in Xenopus mitotic extracts demonstrates its stability (Fig. 2A). The GST tag si mplified purification of stable cyclin B from bacterial extracts (see $M$ aterials and Methods).

Injection of GST-cyclin B protein into syncytial embryos (cycles 1-7) resulted in arrest of the mitotic cycle surrounding the point of injection (Fig. 2B). Arrested mitoses were not seen with control injections of GST. Many of the arrested mitoses show condensed chromosomes that are segregated partially (Fig. 2 insets). Similarly, the arrest induced by expression of stable cyclin B in later cycles also shows an arrest with the chromosomes neither paired at the metaphase plate nor at the spindle poles (Sigrist et al. 1995a; see also Fig. 3). The amount of injected GST-cyclin B approximated that of endogenous cyclin $B$ as determined by immunoblotting (not shown), thus doubling the amount of total cyclin B. Increasing endogenous cyclin B by eightfold does not result in mitotic arrest (G. Schubiger, pers. comm.). This is consistent with observations after induced expression of normal and stable cyclin constructs during the later cycles; these observations indicate that only the stabilized versions of the cyclins have the capacity to arrest the cycle (Sigrist et al. 1995a; Fig. 3G-L). On the basis of the GST-cyclin B-induced block to mitotic progression, 
A

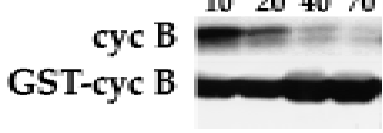

Figure 2. GST-cyclin B causes mitotic arrest. (A) GST-cyclin $B$ is stable in mitotic extracts. In vitro translated ${ }^{35} \mathrm{~S}$-labeled Drosophila cyclin B (cyc B) or a bacterially produced GSTcyclin B fusion (GST - cyc B) was incubated in Xenopus mitotic extracts for various times (minutes) as indicated. Proteins were separated on denaturing gels and cyclin B was detected by autoradiography (cyc B) or by immunoblotting with polyclonal antibodies to GST (GST-cyc B). Although cyc B was degraded in mitotic extracts, GST-cyc B remained stable. (B) Syncytial embryos (0- to 1-hr-old, before cycle 7) were injected with GST-cyclin B, fixed after $30 \mathrm{~min}$, and stained for DNA (red) and $\beta$-tubulin (blue) to visualize microtubules. Nuclei near the site of injection (arrow) are arrested in mitosis and show condensed chromosomes and mitotic spindles. In contrast, in the remainder of the embryo syncytial divisions have progressed and the nuclear density is higher, and nuclei are not arrested in mitosis. Two representative arrested mitotic figures are magnified and shown below.

we infer that destruction of cyclin B, and presumably other mitotic cyclins as well, is required for exit from syncytial mitoses.

Mitotic and nonmitotic nuclei coexist within the syncytial cytoplasm

To reconcile the above observations with the lack of oscillation in total cyclin levels in the early syncytial cycles of the Drosophila embryo and their incomplete destruction during the later syncytial mitoses (Edgar et al. 1994), we suggest that a small pool of cyclins is destroyed during the exit from mitosis. If destruction of cyclins is confined to the locale of each mitotic spindle, local variations in Cdk1 activity may be created that allow syncytial nuclei to exit mitosis in the presence of high levels of Cdk1 activity throughout the rest of the embryo. This proposal predicts that the nuclei of the syncytium would exit mitosis independently of one another. Although the synchrony of the early syncytial cycles is well known, this synchrony is broken in a number of instances that argue for a substantial degree of autonomy among syncytial nuclei.

For example, the products of female meiosis that do not contribute to the zygotic nucleus are not partitioned off as separate cells but remain as nuclei within the syncytium; these "polar bodies" progress through a first mitotic cycle, then arrest in a mitotic-like state with condensed chromosomes, but without a mitotic spindle (Foe et al. 1993; the absence of an associated spindle is presumed to be attributable to the absence of centrosome, which is provided to the zygotic nucleus by the incoming sperm). Importantly, these polar bodies remain in a condensed mitotic state, whereas the nuclei that surround them progress through rapid mitotic cycles. If we

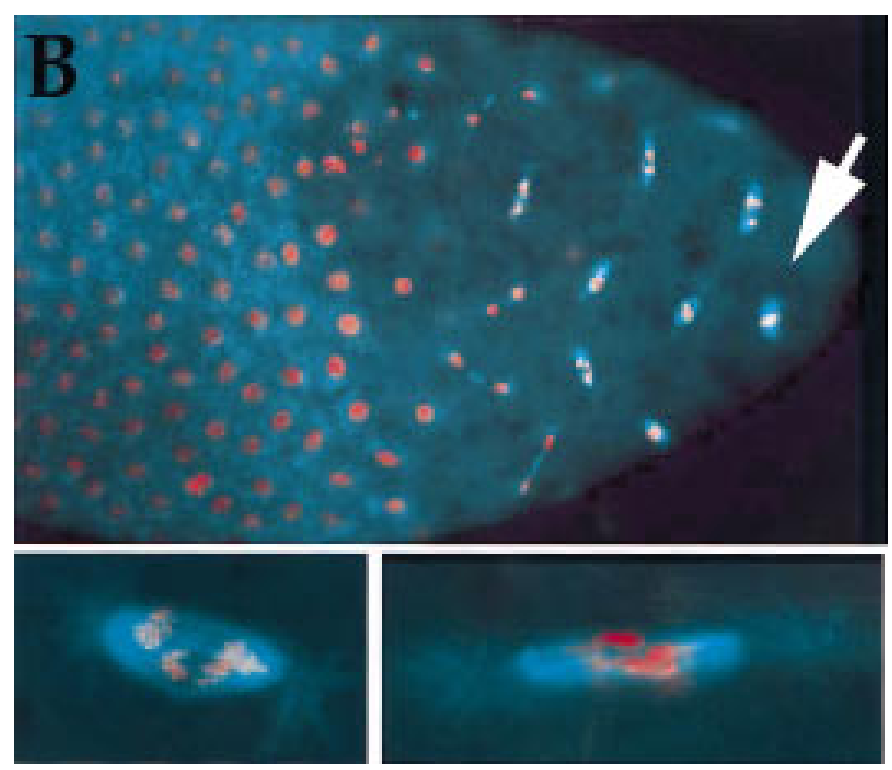

inactivate mitotic kinase $\mathrm{Cdk} 1$ by raising the temperature in a Cdk $1^{\text {ts }}$ mutant, these polar bodies decondense, indicating that the persistent mitotic state requires Cdk1 activity (T.T. Su and P.H. O'Farrell, unpubl.; see below for $\mathrm{Cdk} 1^{\text {ts }}$ experiments). Thus, the mitotic configuration of the polar bodies is consistent with the high levels of Cdk1 kinase. Presumably, when adjacent zygotic mitotic nuclei return to interphase they inactivate Cdk1 locally without eliminating Cdk1 activity in the environment of the polar bodies.

Three other examples suggest that the pol ar bodies are not unique in the autonomy of their cell cycle. Yolk nuclei forgo mitotic events and endoreplicate, whereas the cortical nuclei in the same cytoplasm undergo mitotic divisions (for example, Fig. 30,P). In addition, posterior nuclei that will later become progenitors of germ cells have a division program that first deviates from somatic nuclei when both types of nuclei still share a common cytoplasm (Su et al. 1998). Finally, in Drosophila mutants bearing an abnormally long chromosome, syncytial nuclei are observed to initiate anaphase autonomously (Sullivan et al. 1993). These observations lead us to suggest that exit from mitosis in the syncytial embryo may be under local control.

\section{PH3 signal reflects $\mathrm{Cdk} 1$ activity}

We reasoned that if local variations in $\mathrm{Cdk} 1$ activity are involved in the exit from mitosis in the early syncytial cell cycles, these variations might be detected as local variations in the action of Cdk1. We found that PH3, which can be detected using a specific antibody (Shibata et al. 1990; Bradbury 1992; Ajiro et al. 1996a,b), correlates with Cdk1 activity in Drosophila embryos. H3 phosphorylation is thought to contribute to chromatin condensation during mitosis (Hanks et al. 1983; Th'ng et 


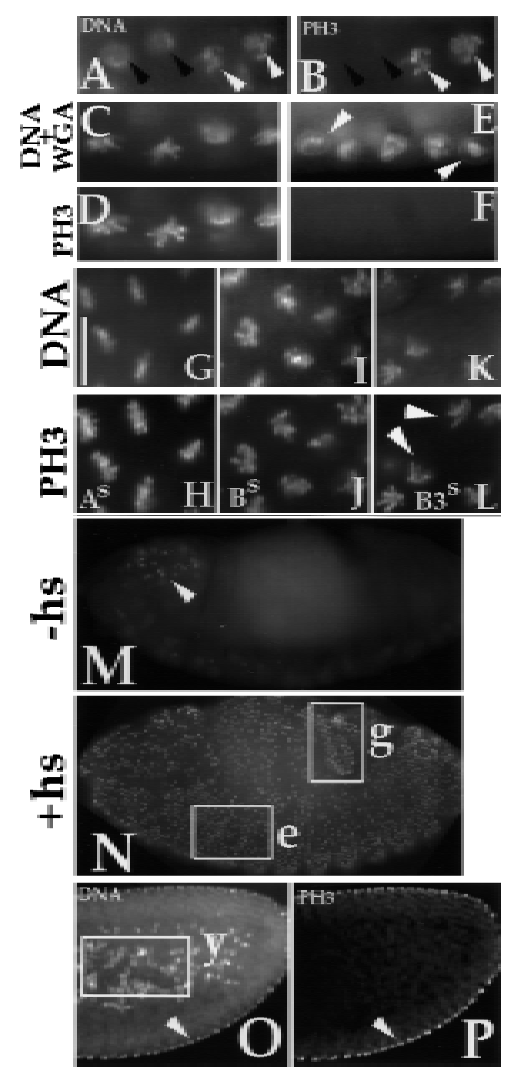

Figure 3. The dependence of $\mathrm{PH} 3$ on $\mathrm{Cdk} 1$ activity in Drosophila embryos. (A,B) PH3 staining at entry into mitosis. Drosophila embryos in division cycle 14-15 were fixed and stained for DN A (A) and PH 3 (B). PH 3 stain is absent in interphase nuclei (black arrowheads) but observed on condensing chromosomes as cells enter prophase (white arrowheads). (C-F) Inactivation of $\mathrm{Cdk} 1$ during a mitotic arrest led to the loss of PH3 signal. Syncytial stage $\mathrm{Cdk} 1^{\text {ts }}$ embryos were arrested in mitosis for $20 \mathrm{~min}$ at the permissive temperature of $18^{\circ} \mathrm{C}$ using colchicine. During this arrest, chromosomes are condensed (C) and stained for PH3 (D). After inactivation of $\mathrm{Cdk} 1^{\text {ts }}$ at $30^{\circ} \mathrm{C}$ for $15 \mathrm{~min}$, chromosomes decondense and become surrounded by the nuclear envel ope $(E$, arrowheads point to wheat germ agglutinin (WGA) staining of nuclear pores) and $\mathrm{PH} 3$ signal is lost (F). (G-L) PH3 during mitotic arrests with nondegradable mitotic cyclins $A\left(A^{s}\right.$ in $\left.G, H\right)$, $B\left(B^{s}\right.$ in $\left.I, J\right)$ or $B 3\left(B 3^{s}\right.$ in $\left.K, L\right)$. After heatinduction of each stable cyclin, embryos were fixed and stained for DNA and PH3. PH 3 stain is present at all three arrests. (M,N) Ectopic expression of Cdk1AF and cyclin A leads to induction of $\mathrm{PH}$ 3. Embryos carrying a heat shock inducible Cdk1AF and cyclin A transgenes were fixed in stages 13-15 (Campos-Ortega and Hartenstein 1985) either directly (M) or $2 \mathrm{hr}$ after heat induction ( $\mathrm{N}$ ) and were stained for $\mathrm{PH} 3$. In M, the normally mitotic cells of the CNS show PH3 staining (arrowhead), but this stain is absent in most other cells, including the epidermis and the gut. In N, PH3 staining is widespread and includes nonmitotic epidermal cells (e) and hindgut cells (g) that are arrested in a postmitosis 16 quiescent phase and are destined to undergo endocycles. The hindgut was identified according to position and morphol ogy viewed by differential interference contrast (DIC) (not shown). (O,P) PH 3 staining of a syncytial embryo demonstrates that cortical nuclei are in a mitotic state and exhibit the $\mathrm{PH} 3$ stain $(\mathrm{O}$, arrowhead), whereas the yolk nuclei ( $\mathrm{y}$, boxed in $\mathrm{N})$ that endoreplicate lack the $\mathrm{PH} 3$ stain. $\mathrm{N}$ ote that the $\mathrm{PH} 3$ signal in internal tissues is detectable by our methods (Su et al . 1988); therefore, the failure of yolk nuclei to stain for $\mathrm{PH} 3$ is unlikely to be attributable to technical reasons. $N$ shows the DNA stain. (A-L) Bar, $10 \mu \mathrm{m}$; (M-O) bar, $40 \mu \mathrm{m}$. al. 1994; Ajiro et al. 1996a,b). In both syncytial and cellularized embryos, phosphorylation of histone $\mathrm{H} 3$ (PH3) signal is absent in interphase and appears on chromosomes as cells enter mitosis (for example, Fig. 3A,B). We carried out three experimental manipulations to test whether the levels of PH3 might be coupled to the activity of Cdk1: (1) inactivation of Cdk1 during mitosis, (2) prolongation of $\mathrm{Cdk} 1$ activity during mitosis, and (3) ectopic induction of Cdk1 activity in interphase.

Inactivation of $\mathrm{Cdk} 1$ during mitosis Ied to a rapid loss of PH3 (Fig. 3C-F). Syncytial embryos bearing a temperature-sensitive version of Cdk1, Cdk $1^{\text {ts }}$ (Sigrist et al. 1995b), were arrested in mitosis using a microtubule depolymerization agent (Fig. 3C,D). Subsequent inactivation of $\mathrm{Cdk} 1^{\text {ts }}$ in these embryos by shifting to the restrictive temperature led to loss of PH3 within 15 min (Fig. 3E,F). Similarly treated wild-type embryos retained PH3 staining as did the control $\mathrm{Cdk}^{\text {ts }}$ embryos that remained at the permissive temperature (not shown). These data indicate that Cdk1 activity is required to maintain $\mathrm{PH} 3$ in mitosis.

Conversely, experimental maintenance of $\mathrm{Cdk} 1$ activity in mitosis led to maintenance of PH3 (Fig. 3G-L). We induced expression of stable versions of cyclins from transgenes under the control of the hsp70 promoter in cellularized embryos (note that heat induction of these transgenes is only possible after cellularization; heat shock before cellularization results in lethality). As shown previously, expression of stable versions of each of the three mitotic cyclins, stable cyclin $A\left(A^{s}\right)$, stable cyclin $B\left(B^{5}\right)$, and stable cyclin $B 3\left(B 3^{5}\right)$, lead to mitotic arrest (Sigrist et al. 1995a). The arrests, however, resemble different stages between metaphase and telophase; $A^{s}$ arrests mitosis with chromosomes at the metaphase plate; $B^{\mathrm{S}}$ arrests mitosis with sister chromosomes separated to varying degrees (early to mid-anaphase-like configuration); and $\mathrm{B}^{\mathrm{s}}$ arrests mitosis with fully separated but fully condensed chromosomes (see arrowheads in Fig. 3L). Importantly, at each arrest point only the stabil ized cyclin persists (Si grist et al. 1995a). PH 3 signal was maintained at all three arrests, suggesting that C dk 1 in complex with any of these mitotic cyclins can maintain $\mathrm{PH} 3$.

Finally, we found that ectopic induction of Cdk1 activity led to induction of PH3. To induce Cdkl activity ectopically, we used heat-induci ble promoters to express Cdk1AF in conjunction with either cyclin A or B. Cdk1AF contains mutations in inhibitory phosphorylation sites (T 14A and Y 15F) and cannot be inhibited by Weel kinase (Sprenger et al. 1997). Consistent with the demonstrated role of inhibitory phosphorylation on $\mathrm{Cdk} 1$ in controlling the progress into mitosis, induction of $\mathrm{Cdk} 1$ with a cyclin triggered $\mathrm{G}_{2}$-arrested cells to enter mitosis and to accumulate $\mathrm{PH}_{3}$ (Su et al. 1998; N. Yakubovich and P.H. O'Farrell, unpubl.). Endocycling cells and $\mathrm{G}_{1}$-arrested cells also responded to induction of Cdk1 and cyclin A by accumulating PH3 (Fig. 3M,N) although these cells did not enter mitosis. Thus, accumulation of $\mathrm{PH} 3$ in response to $\mathrm{Cdk} 1$ activity does not require mitosis. 
Data presented above suggest that $\mathrm{PH} 3$ levels are governed by $\mathrm{Cdk} 1$ activity such that $\mathrm{Cdk} 1$ can induce $\mathrm{PH} 3$ and $\mathrm{Cdk} 1$ is required to maintain $\mathrm{PH} 3$ in syncytial mitoses. At present we do not know whether Cdk1 acts directly on histone H3 in Drosophila.

Localized loss of PH3 signal at exit

from syncytial mitoses

A more detail ed analysis of $\mathrm{PH} 3$ staining upon exit from mitosis reveals an unexpected feature of $\mathrm{PH} 3$ loss during the syncytial cycles. Immunostaining of syncytial embryos for $\mathrm{PH} 3$ demonstrates that chromosomes in metaphase, early anaphase, and mid-anaphase show $\mathrm{PH} 3$ stain al ong their length (Fig. 4A, top two mitotic figures; data not shown). As anaphase progresses, loss of $\mathrm{PH} 3$ begins at the kinetochore regions of chromosomes (Fig. $4 \mathrm{~A}$, the third mitotic figure) and continues as chromosomes decondense in tel ophase (Fig. 4A, bottom mitotic figure). Such local gradients of $\mathrm{PH} 3$ are seen in syncytial mitoses from at least cycle 4 (M 4; the earliest we have anal yzed; M 6 is shown in Fig. 4B) up to and including the last syncytial mitosis, M13 (Fig. 4C), although the PH3 gradient appears increasingly shal lower as nucl ear cycles progress. The localized loss of H3 phosphorylation during anaphase demonstrates that nonuniform conditions occur during exit from syncytial mitosis. A local gradient of kinase activity or a local gradient of phosphatase activity, or a combination of both, could result in the gradient of PH3 staining we observed. Given the strict correlation between $\mathrm{PH} 3$ and $\mathrm{Cdk} 1$ activity in Drosophila embryos described above, we suggest that a likely basis for the localized loss of $\mathrm{PH} 3$ is a localized decline in Cdk1 activity. The localized loss of $\mathrm{PH} 3$ is blocked by injection of the 13-110 peptide (not shown), suggesting that proteolysis contributes to local loss of Cdk1 activity and PH3.
Localized Cdk1 inactivation would explain how syncytial nuclei are able to exit mitosis despite the presence of cyclins and Cdkl activity in the rest of the embryo. Our data are consistent with the degradation of a small and local pool of cyclins, generating a local decline in Cdk1 activity. This would explain the ability of an inhibitor of the APC and a stabilized cyclin to block exit from mitosis during these syncytial cycles.

A priori we might have expected that exit from mitosis requires the complete elimination of cyclins in the environment of the mitotic figures. However, there are indications that any loss of cyclin that might occur is more limited. Staining for cyclins A and B during the syncytial cycles reveal ed strikingly nonuniform distributions, but did not detect a general disappearance of cyclins in the region of anaphase spindles (Maldonado-Codina and Glover 1992). The gradient of PH3 on anaphase chromosomes in syncytial mitoses is consistent with dephosphorylation events originating at the kinetochore regi on or at the spindle pole. We suggest that changes in protein degradation and phosphorylation status of specific components within the mitotic spindle can guide exit from mitosis.

Localized changes in the phosphorylation state of kinetochore proteins have been implicated previously in mitotic spindle function in insect cells ( $\mathrm{N}$ icklas et al. 1995, N icklas 1997). In addition, certain components of the ubiquitin-mediated degradation pathways are localized to the kinetochore region and at the centrosomes (Tugendreich et al. 1995; Bai et al. 1996; Connelly and Heiter 1996; Jorgensen et al. 1998). These and the observations presented here lead us to suggest that mitotic proteolytic activities may also be localized at the kinetochore region or the centrosomes and contribute to mitotic progression. Such local proteolysis of cyclins and inactivation of Cdk1 could explain the local gradient in $\mathrm{PH} 3$ loss we observed in syncytial embryos.
Figure 4. $\mathrm{PH} 3$ staining at exit from mitoses. Wild-type Drosophila embryos were fixed and stained for DNA (red) and PH3 (green). Colocalization of red and green signals result in yellow. $\mathrm{PH} 3$ disappears in a gradient on chromosomes at exit from syncytial mitoses (A-C), but this gradient is not readily apparent in cellular blastoderm mitoses (D). (A) A composite of mitotic figures from syncytial mitoses $10 / 11$ is shown to depict the progression from anaphase (a) to tel ophase ( $\mathrm{t}$ ). Anaphase-tel ophase progression is characterized by a greater separation between kinetochore (pol eward) regions of sister chromosomes, lengthening of chromosome arms, and increasing degree of chromosome decondensation. Loss of $\mathrm{PH} 3$ begins at the kinetochore regions of chromosomes in late anaphase/tel ophase. $\mathrm{PH} 3$ gradients are seen in M 6 (B) and in M 13 (C). Only slight color gradients are seen in late anaphase figures of M 14 (D, brackets show two pairs of late anaphase/telophase chromosomes) and these gradations appear to be caused by nonhomogenous Hoechst 33258 staining of DN A rather than by nonhomogenous PH3 stain (see Fig. 5). Bar (A) $10 \mu \mathrm{m}$; (B-D) $20 \mu \mathrm{m}$.

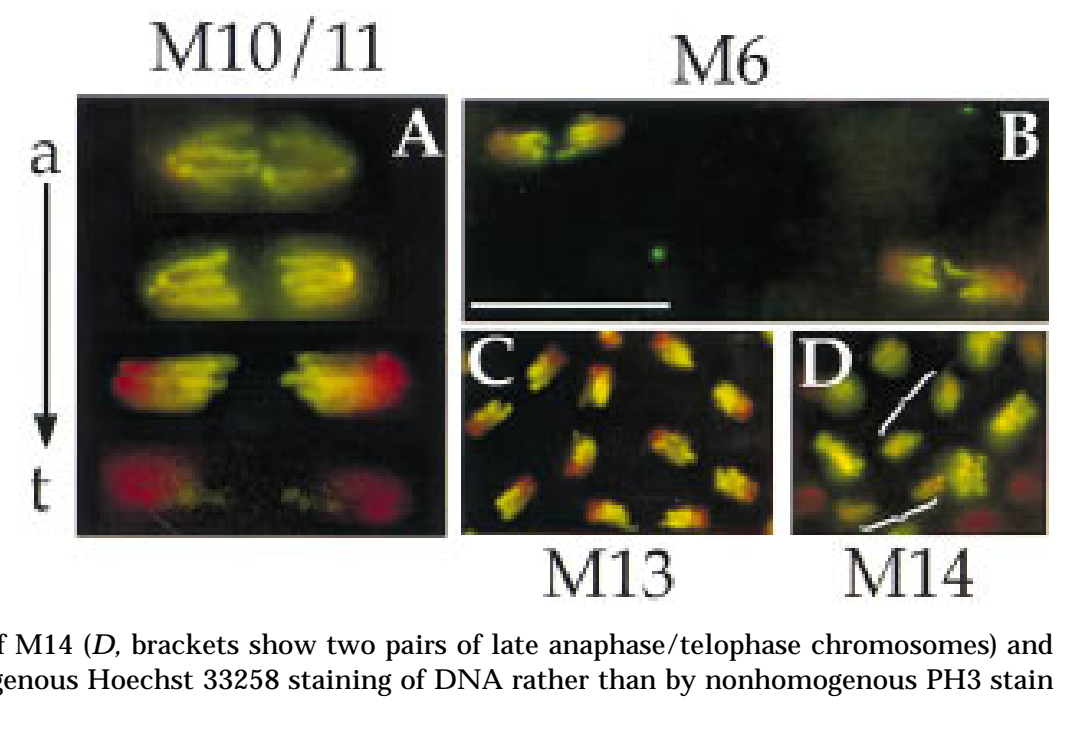


$\mathrm{PH} 3$ loss is near uniform in mitoses of cellularized embryos

At the conclusion of the last syncytial division (M 13), the embryo undergoes many changes associated with the maternal to zygotic transition [(MZT; similar to midblastula transition (MBT) of vertebrates], including celIularization and the transition from the maternal to zygotic control of the cell cycle (Foe et al. 1993). Of these changes, the most pertinent to this work are the changes in cycl in degradation; whereas no mitotic cyclin destruction is detected before the ninth mitosis, at each subsequent mitosis a larger and larger fraction of cyclins $A$ and $B$ are degraded until, subsequent to the MZT, cyclin destruction at mitosis 14 appears to be complete (Lehner and O'Farrell 1989, 1990; Edgar et al. 1994; Sigrist et al. 1995a). Paralleling these changes, there is a change in histone $\mathrm{H} 3$ dephosphorylation at exit from mitosis. The gradient of $\mathrm{PH} 3$ staining al ong the anaphase chromosomes becomes less marked in the later syncytial divisions, and then in M 14, $\mathrm{PH} 3$ persists on chromosomes throughout anaphase and di sappears approximately uniformly from chromosomes in telophase (Figs. 4D and 5). Although the two-color images in Figure 4D suggest that a slight gradient might persist into mitosis 14, the separate staining for DN A shown in Figure 5 suggests that uneven color ratios in $M 14$ largely results from the nonuniformity of the Hoechst 33258 staining of the DNA. $\mathrm{PH} 3$ staining in the two remaining embryonic mitoses in the epidermis, M 15 and M 16, is similar to that of M 14 (data not shown).

What might underlie the gradual change in histone $\mathrm{H} 3$ dephosphorylation as divisions progress? We suggest that it reflects the change in cyclin destruction. As suggested above, the incomplete cyclin destruction and local loss of $\mathrm{PH} 3$ during the syncytial cycles might be attributable to localization of cyclin destruction machinery, in which case the delocalization of this machinery in cycle 14 might result in the complete cyclin destruction and near-uniform loss of PH3 seen during M 14. Re-

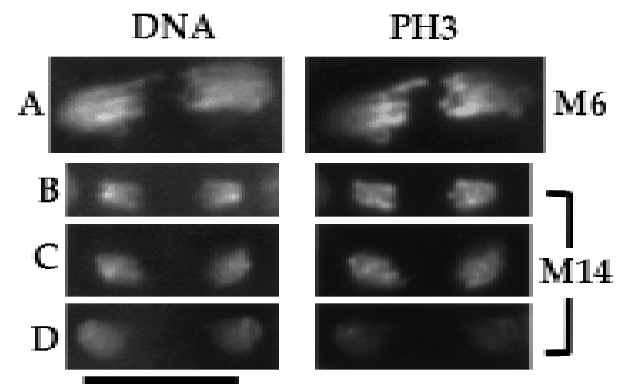

Figure 5. PH3 staining disappears uniformly as cellular blastoderm nuclei exit mitosis. Wild-type Drosophila embryos were fixed and stained for DN A and PH3 as indicated. (B-D) A progression from late anaphase through telophase of $M$ 14. These mitotic chromosomes are from the same embryo and have been processed identically. A decline in $\mathrm{PH} 3$ signal from $B$ to $D$ is evident but does not occur in a gradient as in syncytial mitoses (to allow comparison, A shows M 6 chromosomes from Fig. 4B). Bar, $10 \mu \mathrm{m}$. gardless of the mechanism, it is noteworthy that the mode of histone $\mathrm{H} 3$ dephosphorylation changes at the time of profound transitions that comprise the MZT. Hence, the manner in which exit from mitosis is executed may be developmentally regulated, just as the entry into mitosis is devel opmental ly regulated (Edgar et al. 1994). We suggest that localized cyclin destruction is a specialization of early syncytial cycles that allows local control of the exit from mitosis despite the absence of cell membranes and that this specialization is lost in concert with cellularization.

\section{Materials and methods}

\section{Fly stocks}

The $C d k 1^{\text {ts }}(A 171 T)$ stock and stocks harboring heat shock-inducible stable cyclins Cdk1AF and cyclins A and B have been described before (Sigrist et al. 1995a; Sprenger et al. 1997).

\section{Embryo-staining procedures}

For antibody staining embryos were fixed for $20 \mathrm{~min}$ in PBS $+10 \%$ formal dehyde or for $5 \mathrm{~min}$ in $37 \%$ formaldehyde (injected embryos, see bel ow), using standard procedures. DN A was stained with $10 \mu \mathrm{g} / \mathrm{ml}$ bizbenzamide (Hoechst 33258). FITC-labeled wheat germ agglutinin (WGA) was used at 500 $\mathrm{ng} / \mathrm{ml}$ (Molecular Probes). PH3 was detected with a purified rabbit polyclonal antibody (1:1000 dilution) against the peptide ARKSTGGKAPRKQL (present in three Drosophila H 3 variants) in which $S$ was phosphorylated (Upstate Biotechnologies). $\beta$ Tubulin antibodies (Amersham) were used at 1:100 dilution. Primary antibodies were probed with rhodamine or FITC-conjugated secondary antibodies diluted 1:500 (Jackson).

Injection

Expression and purification of 13-110 (amino terminus of sea urchin cyclin B) and 13-110* (identical to 13-110 except for two-point mutations described in the text) peptides was performed as previously described (Holloway et al. 1993), except for a modification of the column chromatography. Supernatant after $85^{\circ} \mathrm{C}$ incubation was applied to a phosphocellulose column equilibrated in $20 \mathrm{~mm}$ PIPES (pH 6.5), $50 \mathrm{~mm} \mathrm{~N} \mathrm{aCl}$. The column was washed sequentially with three column volumes 100, 300, and $500 \mathrm{~mm}$ of $\mathrm{NaCl}$ in $20 \mathrm{~mm}$ PIPES (pH 6.5). The peptide eluted predominantly in the $300 \mathrm{~mm} \mathrm{~N}$ aCl step elution and was $>90 \%$ pure by Coomassie blue staining on a SDS gel. Fractions from the $300 \mathrm{~mm} \mathrm{NaCl}$ elution were pooled, concentrated using a Centriprep-3 (Amicon), dialyzed overnight at $4^{\circ} \mathrm{C}$ against 5 $\mathrm{mm} \mathrm{NaCl}, 0.1 \mathrm{~mm} \mathrm{NaHPO}{ }_{4}(\mathrm{pH} 7.7$ ) injection buffer, and concentrated again to a final concentration of $50 \mathrm{mg} / \mathrm{ml}$ using a Microcon-3 (Amicon).

Sevelin embryos (0-1 hr) were prepared and microinjected, as described previously (Su et al. 1997), with $5 \mathrm{mg} / \mathrm{ml}$ peptide in 5 mм NaCl, $0.1 \mathrm{~mm} \mathrm{NaHPO}_{4}$ (pH 7.7). Estimated injection volume was between $2 \%$ and $5 \%$ of the total volume of the embryo. Injected embryos were aged at $18^{\circ} \mathrm{C}$ for $30 \mathrm{~min}$ before fixing. The GST-cyclin B protein used in this study was a gift from Douglas Kellogg (University of California, Santa Cruz) and the GST was kindly provided by Danesh M oazed (University of California, San Fransisco). GST-cyclin B was injected at $6 \mathrm{mg} /$ $\mathrm{ml}$ and GST at $12 \mathrm{mg} / \mathrm{ml}$ in $25 \mathrm{~mm}$ HEPES (pH 7.4), $125 \mathrm{~mm}$ $\mathrm{KCl}, 10 \%$ glycerol. Injected embryos were aged at $18^{\circ} \mathrm{C}$ for 30 min, fixed for $5 \mathrm{~min}$ in 37\% formal dehyde, devitellinized manu- 
ally as described previously (Su et al. 1997), and stained with anti-tubulin antibody (1:100, Amersham) and propidium iodide or Hoechst 33258 to visualize DNA.

\section{Miscellaneous procedures}

To arrest $C d k 1^{\text {ts }}$ embryos in mitosis, syncytial stage embryos were permeabilized according to standard procedures and incubated in Schneider's medium containing $500 \mu \mathrm{g} / \mathrm{ml}$ colchicine (Sigma). To arrest with stable cyclins, 2- to 4-hr-old embryos were exposed to $37^{\circ} \mathrm{C}$ for $30 \mathrm{~min}$, allowed to recover at room temperature for $2 \mathrm{hr}\left(\mathrm{A}^{\mathrm{s}}\right.$ and $\left.\mathrm{B}^{\mathrm{s}}\right)$ or $1 \mathrm{hr}\left(\mathrm{B}^{\mathrm{s}}\right)$ to achieve maximal arrest per embryo, and fixed as above. To induce Cdk1AF + cyclin A or B, 10- to 12-hr-old embryos were heatshocked as above and fixed after $2 \mathrm{hr}$ rest at room temperature.

Incubation in Xenopus mitotic extracts were performed as described previously (King et al. 1996b).

Division cycle in syncytial embryos was determined from nuclei number ( $n=2$ for cycle $2 ; n=4$ for cycle 3 , etc.) and nuclear location with respect to the embryo surface (in embryo interior through cycle 8 , migrate during cycle 9 , and reach the surface at the end of cycle 9; Foe et al. 1993).

\section{Acknowledgments}

We thank D. Kellogg for GST-cyclin B, D. Moazed for GST protein, the A.W. M urray laboratory for pepti de expression plasmids, Alex Szidon for help with the peptide purification, and C. Detweiler and M. M axon for critical reading of the manuscript. F.S. was supported by the Human Frontier Science Program (H.F.S.P.) and the M ax-Planck Society, S.D.C. was supported by a Centennial fellowship from the Medical Research Council of Canada, and T.T.S. was supported by a Herbert Boyer postdoctoral fellowship. This work was supported by $\mathrm{N}$ ational Institutes of Health grant GM 37193 to P.H.O.

The publication costs of this article were defrayed in part by payment of page charges. This article must therefore be hereby marked "advertisement" in accordance with 18 USC section 1734 solely to indicate this fact.

\section{References}

Ajiro, K., H. Yasuda, and H. Tsuji. 1996a. Vanadate triggers the transition from chromosome condensation to decondensation in a mitotic mutant (tsTM 13) inactivation of p34cdc2/ $\mathrm{H} 1$ kinase and dephosphorylation of mitosis-specific histone H3. Eur. J. Biochem. 241: 923-930.

Ajiro, K., K. Yoda, K. Utsumi, and Y. Nishikawa. 1996b. Alteration of cell cycle-dependent histone phosphorylations by okadaic acid. Induction of mitosis-specific H3 phosphorylation and chromatin condensation in mammalian interphase cells. J. Biol. Chem. 271: 13197-13201.

Bai, C., P. Sen, K. Hofmann, L. Ma, M. Goebl, J.W. Harper, and S.J. Elledge. 1996. SKP1 connects cell cycle regulators to the ubiquitin proteolysis machinery through a novel motif, the F-box. Cell 86: 263-274.

Bradbury, E.M. 1992. Reversible histone modifications and the chromosome cell cycle. Bioessays 14: 9-16.

Campbell, S.D., F. Sprenger, B.A. Edgar, and P.H. O'Farrell. 1995. Drosophila Weel kinase rescues fission yeast from $\mathrm{mi}$ totic catastrophe and phosphorylates Drosophila Cdc2 in vitro. Mol. Biol. Cell 6: 1333-1347.

Campos-Ortega, J.A. and V. Hartenstein. 1985. The embryonic development of Drosophila melanogaster. Springer-Verlag, Berlin, Germany.
Cohen-Fix, O., J.M. Peters, M.W. Kirschner, and D. Koshland. 1996. Anaphase initiation in Saccharomyces cerevisiae is controlled by the APC-dependent degradation of the anaphase inhibitor Pdslp. Genes \& Dev. 10: 3081-3093.

Connelly, C. and P. Heiter. 1996. Budding yeast SKP1 encodes an evolutionarily conserved kinetochore protein required for cell cycle progression. Cell 86: 275-285.

Edgar, B.A., F. Sprenger, R.J. Duronio, P. Leopold, and P.H. O'Farrell. 1994. Distinct molecular mechanisms regulate cell cycle timing at successive stages of Drosophila embryogenesis. Genes \& Dev. 8: 440-452.

Foe, V.E., G.M. Odell, and B.A. Edgar. 1993. Mitosis and morphogenesis in the Drosophila embryo. In The development of Drosophila melanogaster (ed. M. Bate and A. Martinez Arias), pp. 149-300. Cold Spring Harbor Laboratory Press, Cold Spring Harbor, NY.

Follette, P.J. and P.H. O'Farrell. 1997. Cdks and the Drosophila cell cycle. Curr. Opin. Genet. Dev. 7: 17-22.

Funabiki, H., H. Yamano, K. Kumada, K. Nagao, T. Hunt, and M. Y anagida. 1996. Cut2 proteolysis required for sister-chromatid separation in fission yeast. Nature 381: 438-441.

Gallant, P. and E.A. Nigg. 1992. Cyclin B2 undergoes cell cycledependent nuclear translocation and, when expressed as a non-destructible mutant, causes mitotic arrest in HeLa cells. J. Cell Biol. 117: 213-224.

Glotzer, M., A.W. Murray, and M.W. Kirschner. 1991. Cyclin is degraded by the ubiquitin pathway. Nature 349: 132-138.

Hanks, S.K., L.V. Rodriguez, and P.N. Rao. 1983. Relationship between histone phosphorylation and premature chromosome condensation. Exp. Cell Res. 148: 293-302.

Holloway, S.L., M. Glotzer, R.W. King, and A.W. M urray. 1993. Anaphase is initiated by proteolysis rather than by the inactivation of maturation-promoting factor. Cell 73: 13931402.

Jorgensen, P.M., E. Brundell, M. Starborg, and C. Hoog. 1998. A subunit of the anaphase-promoting complex is a centromereassociated protein in mammalian cells. Mol. Cell. Biol. 18: $468-476$.

Juang, Y.L., J. Huang, J.M. Peters, M.E. McLaughlin, C.Y. Tai, and D. Pellman. 1997. APC-mediated proteolysis of Asel and the morphogenesis of the mitotic spindle. Science 275: 1311-1314.

King, R.W., J.M. Peters, S. Tugendreich, M. Rolfe, P. Hieter, and M.W. Kirschner. 1995. A 205 complex containing CDC27 and CDC16 catalyzes the mitosis-specific conjugation of ubiquitin to cyclin B. Cell 81: 279-288.

King, R.W., R.J. Deshaies, J.M. Peters, and M.W. Kirschner. 1996a. How proteolysis drives the cell cycle. Science 274: 1652-1659.

King, R.W., M. Glotzer, and M.W. Kirschner. 1996b. M utagenic analysis of the destruction signal of mitotic cyclins and structural characterization of ubiquitinated intermediates. Mol. Biol. Cell. 7: 1343-1357.

Knoblich, J.A. and C.F. Lehner. 1993. Synergistic action of Drosophila cyclins A and B during the G2-M transition. EMBO J. 12: $65-74$.

Lehner, C.F. and P.H. O'Farrell. 1989. Expression and function of Drosophila cyclin A during embryonic cell cycle progression. Cell 56: 957-968.

- - - 1990. The roles of Drosophila cyclins A and B in mitotic control. Cell 61: 535-547.

M aldonado-Codina, G. and D.M. Glover. 1992. Cyclins A and B associate with chromatin and the polar regions of spindles, respectively, and do not undergo complete degradation at anaphase in syncytial Drosophila embryos. J. Cell Biol. 116: 967-976. 
Minshull, J., A. Straight, A.D. Rudner, A.F. Dernburg, A. Belmont, and A.W. Murray. 1996. Protein phosphatase 2A regulates M PF activity and sister chromatid cohesion in budding yeast. Curr. Biol. 6: 1609-1620.

Murray, A.W., M.J. Solomon, and M.W. Kirschner. 1989. The role of cyclin synthesis and degradation in the control of maturation promoting factor activity. Nature 339: 280-286.

Nasmyth, K. 1996. At the heart of the budding yeast cell cycle. Trends Genet. 12: 405-412.

Nicklas, R.B. 1997. How cells get the right chromosomes. Science 275: 632-637.

Nicklas, R.B., S.C. Ward, and G.J. Gorbsky. 1995. Kinetochore chemistry is sensitive to tension and may link mitotic forces to a cell cycle checkpoint. J. Cell Biol. 130: 929-939.

Pu, R.T. and S.A. Osmani. 1995. Mitotic destruction of the cell cycle regulated N IM A protein kinase of Aspergillus nidulans is required for mitotic exit. EMBO J. 14: 995-1003.

Schwab, M., A.S. Lutum, and W. Seufert. 1997. Y east Hct1 is a regulator of Clb2 cyclin proteolysis. Cell 90: 683-693.

Shibata, K., M. Inagaki, and K. Ajiro. 1990. M itosis-specific histone $\mathrm{H} 3$ phosphorylation in vitro in nucleosome structures. Eur. J. Biochem. 192: 87-93.

Sigrist, S., H. Jacobs, R. Stratmann, and C.F. Lehner. 1995a. Exit from mitosis is regulated by Drosophila fizzy and the se quential destruction of cyclins $A, B$ and B3. EMBO J. 14: 4827-4838.

Sigrist, S., G. Ried, and C.F. Lehner. 1995b. Dmcdc2 kinase is required for both meiotic divisions during Drosophila spermatogenesis and is activated by the Twine/cdc25 phosphatase. Mech. Dev. 53: 247-260.

Sprenger, F., N. Yakubovich, and P.H. O'Farrell. 1997. S-phase function of Drosophila cyclin A and its downregulation in G1 phase. Curr. Biol. 7: 488-499.

Su, T.T., N. Yakubovich, and P.H. O'Farrell. 1997. Cloning of Drosophila MCM homologs and analysis of their require ment during embryogenesis. Gene 192: 283-289.

Su, T.T., S.D. Campbell, and P.H. O'Farrell. 1998. Cell cycle program in drosophila germ cells. Dev. Biol. (in press).

Sudakin, V., D. Ganoth, A. Dahan, H. Heller, J. Hershko, F.C. Luca, J.V. Ruderman, and A. Hershko. 1995. The cyclosome, a large complex containing cyclin-selective ubiquitin ligase activity, targets cyclins for destruction at the end of mitosis. Mol. Biol. Cell. 6: 185-197.

Sullivan, W., D.R. Daily, P. Fogarty, K.J. Yook, and S. Pimpinelli. 1993. Delays in anaphase initiation occur in individual nuclei of the syncytial Drosophila embryo. Mol. Biol. Cell. 4: 885-896.

Surana, U., A. Amon, C. Dowzer, J. McGrew, B. Byers, and K. N asmyth. 1993. Destruction of the CDC28/CLB mitotic kinase is not required for the metaphase to anaphase transition in budding yeast. EMBO J. 12: 1969-1978.

Th'ng, J.P., X.W. Guo, R.A. Swank, H.A. Crissman, and E.M. Bradbury. 1994. Inhibition of histone phosphorylation by staurosporine leads to chromosome decondensation. J. Biol. Chem. 269: 9568-9573.

Tugendreich, S., J. Tomkiel, W. Earnshaw, and P. Hieter. 1995. $\mathrm{CDC} 27 \mathrm{Hs}$ colocalizes with $\mathrm{CDC} 16 \mathrm{Hs}$ to the centrosome and mitotic spindle and is essential for the metaphase to anaphase transition. Cell 81: 261-268.

van der Velden, H.M. and M.J. Lohka. 1993. Mitotic arrest caused by the amino terminus of Xenopus cyclin B2. Mol. Cell. Biol. 13: 1480-1488.

Yamano, H., J. Gannon, and T. Hunt. 1996. The role of proteolysis in cell cycle progression in Schizosaccharomyces pombe. EMBO J. 15: 5268-5279. 


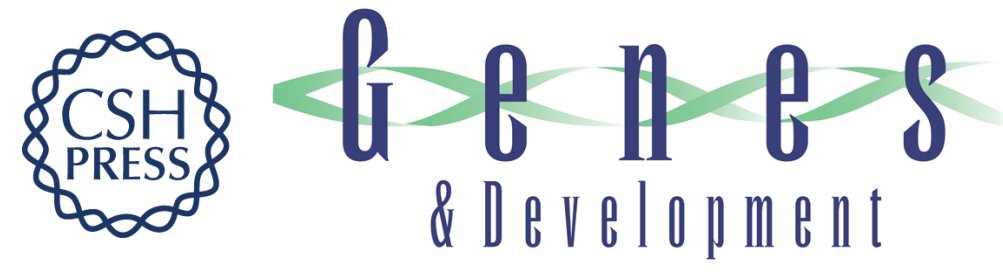

\section{Exit from mitosis inDrosophila syncytial embryos requires proteolysis and cyclin degradation, and is associated with localized dephosphorylation}

Tin Tin Su, Frank Sprenger, Paul J. DiGregorio, et al.

Genes Dev. 1998, 12:

Access the most recent version at doi:10.1101/gad.12.10.1495

\section{References This article cites 42 articles, 15 of which can be accessed free at: http://genesdev.cshlp.org/content/12/10/1495.full.html\#ref-list-1}

\section{License}
Email Alerting Receive free email alerts when new articles cite this article - sign up in the box at the top Service right corner of the article or click here.

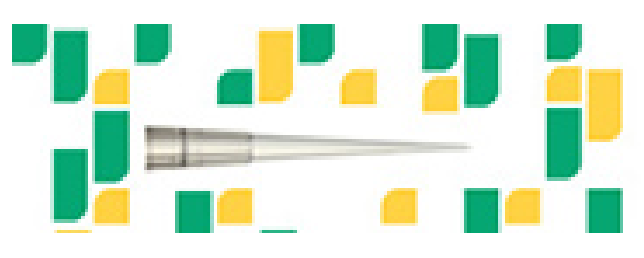

